\title{
TIRED TRUMP oder: Die Ermüdung der Theorie
}

\author{
Simon Strick
}

Andy walking, Andy tired, Andy take a little snooze.

David Bowie, „Andy Warhol“, Hunky Dory (1971)

\begin{abstract}
Als sein Vater zum Schock der Weltöffentlichkeit zum Präsidenten der Vereinigten Staaten von Amerika gewählt wurde, überkam Barron Trump eine schwere Müdigkeit. Auf dem Podium, linkerhand des gestikulierenden Ungetüms, hielt der zehn Jahre alte Junge mit Mühe die Augen offen. Das Getriebe der demokratischen Ordnung knirschte, und Barron unterdrückte weltweit sichtbar ein Gähnen (Abb. 1).

Wie Heiner Müller, der einmal auf die Frage, wie er die historische Rolle eines bestimmten Politikers einschätze, lapidar sagte: „Der ist nur der Kuckuck auf der Kuckucksuhr", ${ }^{\text {s }}$ schien Barron vom Sieg seines Vaters gelangweilt. Was wusste Barron Trump, dass er fast einschlief, während die Welt den Zusammenbruch der amerikanischen Demokratie atemlos verfolgte? Was ist die Kuckucksuhr, deren Türchen regelmäßig die Schreie des Donald J. Trump in die Welt entlässt?

Folgen wir Avital Ronells Stupidity (2002) und mit ihr Marx, gibt die Lautstärke den Hinweis auf den Apparat: Trump ist derzeit sichtbarster Grüßaugust und Kühlerfigur der „Geschichtsmacht Dummheit“ (323). Ronells Monografie - ihre Einleitung liest sich wie eine akkurate Beschreibung der letzten zwei Jahre - begreift das Objekt als Soundwall, die alle Störgeräusche im eigenen Lärm
\end{abstract}

\footnotetext{
${ }^{1}$ Trump stellt ein Verwirrungsmoment dar, das uns eint. Die Verwirrung führt dazu, dass sich viele Auslassungen zum Thema in Gesprächen, Vorträgen und Bemerkungen mitteilen. Der wissenschaftliche Aufsatz bietet nicht immer Platz und Form für dieses ungeordnete Denken, das aber wichtig zur Annäherung an den Topos ist. Aus diesem Grund zitiere ich hier u. a. Gehörtes und Erinnertes. Zur Kenntlichmachung gebe ich bei diesen Zitaten Vor- und Zunamen der Urheber*innen an. Aussagen des Wortgewitters Trump werden nicht nachgewiesen.
}

\author{
S. Strick $(\bowtie)$ \\ Berlin, Deutschland \\ E-Mail: strick@gsnas.fu-berlin.de
}




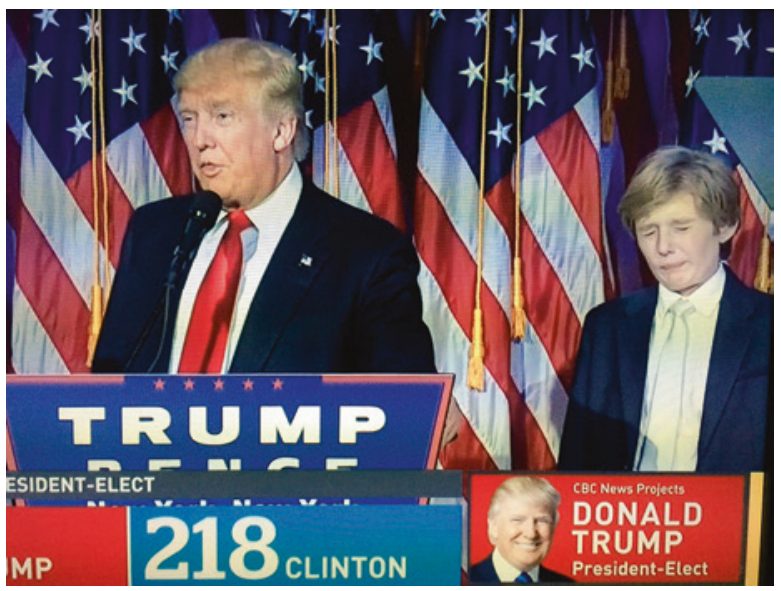

Abb. 1 Tired Barron

ertränkt: „[...] stupidity, purveyor of self-assured assertiveness, mutes just about everything that would seek to disturb its impervious hierarchies" (ebd.: 3). Eine Kritik der Dummheit könnte stören, säuft aber im Lärm ab. Der Präsident zwitschert bzw. twittert in diesem Sinn nicht; Trump is all-noise, oder ,sound and fury and garble“, wie Lauren Berlant (2016) schreibt. Mit Shakespeare und Social Media ließe sich sagen: „Trump's is an idiot's tale, Twitter amplified, spell-check disabled.“

\section{The Idiot President}

Amerikanische Öffentlichkeiten haben sich intensiv mit der sprachlichen Müllproduktion des Donalds beschäftigt, ohne zu ermüden. Anhand des ,Garble' sei der ,Idiot‘ zu entlarven. Seit den republikanischen Vorwahlen analysierten Journalist*innen und Wissenschaftler*innen den Irrwitz Trumpschen Satzbaus, seinen semantischen Dilettantismus. Man stellte das Sprachniveau der politischen Botschaft fest und diagnostizierte Kulturverfall: „For grammar, we see that the level for Donald Trump is significantly lower, at grade 5" (Schumacher/Eskenazi 2016). Eine andere Form der Regression in Angriff nehmend, fanden sich 27 Psycholog*innen zu der Ferndiagnose zusammen, dass Trump laut DSM an „malignant narcissism“, „clinical sociopathy“ und ,antisocial personality disorder" leide (vgl. Lee 2017: 51 f.). Trumps Unfähigkeit, das Wort ,anonymous“ fehlerfrei auszusprechen, am 07.09.2018 in Montana dokumentiert, war das jüngste Indiz zur Überführung des intellektuell eingeschränkten und/oder geistig verwirrten Präsidenten. Wiederholt wird das Notskalpell des 25th Amendment diskutiert, um die Impotenz politischer Geistesmacht rechtskonform zu beseitigen: „Whenever the President [...] is unable to discharge the powers and duties of his office", heißt es dort schmucklos phallozentrisch. Kommentare überbieten sich mit 
abwertenden Einschätzungen der präsidialen Gehirnleistung. Kondensat ist jene Enthüllung, die Bob Woodward dem ehemaligen ,White House Chief of Staff John Kelly nachsagt: „He's an idiot. It's pointless to try to convince him of anything. He's gone off the rails“" (zit. n. Paschal 2018). Was enthüllt die Hermeneutik des he said, she said?

Nichts. Bereits in Wordsworths Langgedicht The Idiot Boy (1799) spielt der Idiot ohne Kostüm und auf kalt ausgeleuchteter Bühne: „And the sun did shine so cold!“ Seine Weltverzerrung muss bzw. kann nicht interpretativ entschlüsselt werden. Am „Burrr Burrr“ von Wordsworths Idioten beißt sich die Literaturwissenschaft denselben Zahn aus, den der politische Kommentar bei Trumps „covfefe“ verliert. Ähnliche Bissschwäche erfuhr feministische und antirassistische Kritik, als sie Trumps „grab'em by the pussy“ oder ,very bad hombres“ aufnahm. Was analysieren? Stupidity gewinnt nicht durch Verstellung oder Maskenspiel, sondern durch schiere Präsenz. Stupidity ist „,what is there“, so Ronell, auch wenn ,[...] it cannot be simply located or evenly scored“ (2002: 3). Es erschien Erfolg versprechend, den Präsidenten als Rassisten, Sexisten oder HONK (Hauptschüler ohne nennenswerte Kenntnisse) zu demaskieren; alas, eine Maske gab es nicht. So wundert es auch nicht, dass eine tief gehende populärkulturelle Aufschlüsselung Trumpscher Performance - Georg Seeßlens Büchlein Trump! POPulismus als Politik - zur Amtsvereidigung im Januar 2017 schon vorlag. Alles war und ist offensichtlich. Erstes Ermüden.

Bleiben wir aber noch bei stupidity. Der Begriff schwankt zwischen Abwertung und medizinischem Befund, Beleidigung und Pathologie. In ihm zeigt sich die Unentschlossenheit, wie dem politischen Gegner entgegenzutreten ist. Ist er lächerlich, oder ist er krank? ${ }^{2}$ Sind wir Ärzt*innen oder haben wir den mental bzw. moral high ground? ,Stupid Trump“ - die invektive Diagnose scheitert auch, weil sie politisch keine Lösungen zeitigt. Seit Januar 2017 ist deutlich, dass Blödheit für nichts disqualifiziert, und dass ,nicht-dumm“ (nicht-sexistisch, nicht-rassistisch, usw.) zu sein weder ein politisches Programm noch Wähler*innengunst impliziert. ,Trumpian stupidity‘ beschreibt eher Marke als Makel, und scheitert, als Argument vorgebracht, an der eigenen problematischen Genealogie:

\footnotetext{
„When did ,stupid“ become a denunciation? Why did we begin to figure the other as stupid? [...] One would have to review the consistent naming of the slave as the nonhuman, the ineducable, in terms of phantasms of calculable intelligence. What has morphed into seemingly less lacerating assertions of stupidity (,shallow, , ,airhead, ",bimbo, ', brain-

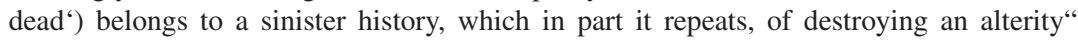
(Ronell 2002: 39 f.).
}

Abseits simpler Ausgrenzung ist das politische Argument gegen einen Präsidenten mit niedrigem IQ, Demenz, klinischer Depression oder Narzissmus schlicht nicht bestimmbar. Die kritischen Disability Studies haben einiges zu sagen zur

\footnotetext{
${ }^{2}$ Eine ähnliche Problemlage untersuchen Sander Gilman und James Thomas in ihrem Buch Are Racists Crazy? (2016).
} 
Abwertung von Menschen durch fiktive Hierarchien der Intelligenz, Normen ,geistiger Gesundheit ', und der mörderischen Geschichte dieser Deklassierungsmechanismen (Rapley 2004). Fliehen wir vor der Dummheit des US-Präsidenten, finden wir uns beim eigenen ableism wieder. Essentialisieren wir Trump auf diese Weise als ,Idiot', bleibt zudem die tiefe Genealogie präsidialer Dämlichkeit unreflektiert, die Jude Davies (2007) bereits zu Zeiten des jüngeren George Bush skizziert hat.

Wir sind also vor eine ähnliche Aufgabe der Begriffsneufindung gestellt wie sie uns von den gegenwärtigen Vertreter*innen der neuen Rechten auferlegt

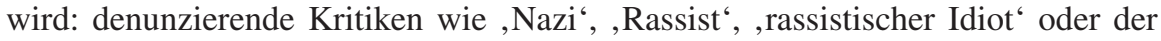
US-amerikanische Sonderfall ,bigot‘ lösen seitens der Alt-Right (Hawley 2017) keine diskursiven Rückzüge wegen Verwundung mehr aus. Nothing hurts. Im ersten Schritt müssten wir zumindest unsere Invektiven aufbohren, sie besser und nuancierter gestalten. Gute Beleidigungen ,treffen': sie zerren einen lieber versteckten Wesenszug hervor, und sprechen eine Anerkennung (recognition) wider Willen aus. Die gute Beleidigung ist Geschenk und geteilte Erkenntnis, nicht halbmedizinischer Befund des Offenbaren. Das Online-Portal Jezebel.com hat Trump zum 70. Geburtstag einen Katalog von 70 richtungsweisenden Beleidigungen überreicht (Rothkopf 2016), aus denen ich - Heuristik der Invektive - Nicht-Intuitives ableiten möchte. Die Titel sind zu lesen nach dem Prinzip: Donald Trump is...

\section{Your Shitty Racist Uncle}

Viele Befunde sprechen derzeit von einer ,Rückkehr des starken Mannes“ (Georg Seeßlen), versinnbildlicht durch Trumps, dezisionistische Männlichkeit‘ (Paula Villa) und seine ,Hypermaskulinität‘ (Sieglinde Lemke). Die unverstellte Idiotie Trumps deutet dagegen dessen irgendwie nicht-hegemoniale Männlichkeit an (Conell 2005); seine Biografie - als reich geborener und ewig unvollendeter Sohn des Self-Made-Patriarchen Fred - tut ihr Übriges. Überaus sichtbar ist seine unklassische Männlichkeit im ,Gesetz des Vaters', wie es von The Don rituell desavouiert wird: Trumps vermeintliche Autorität beschwört sich stets als Eigensabotage, nicht durch patriarchale Absolutheit. Performative Widersprüche legt er selbst im ,selbstdurchstreichenden“ Eigenlob vor - „I have the best brain“, „I'm a very stable genius“, usw. - oder andere üben sich im dekonstruktiven Politik-Dada. Hier ein Auszug aus Sarah Palins denkwürdigem Manifest für eine poésie concrète, ihrem endorsement für Trump während der primaries:

„Yeah, our leader is a little bit different. He's a multi-billionaire. Not that there's anything wrong with that. But, it's amazing, he is not elitist at all. Oh, I just hope you all get to know him more and more as a person, and a family man. What he's been able to accomplish, with his um, it's kind of this quiet generosity. Yeah, maybe his largess kind of, I don't know, some would say gets in the way of that quiet generosity, and, uh, his compassion, but if you know him as a person and you'll get to know him more and more, 
you'll have even more respect. [...] And yes, as a multi-billionaire, we still root him on, because he roots us on" (CBSN 2016).

Palins sehenswert surreale Wahlempfehlung (vgl. Schilling 2016) umarmt den Kandidaten mit der Grillzange. Sie beschwört Trump ${ }^{\mathrm{TM}}$ nicht als den natürlichen Nom-du-Père (Lacan); sein Name verkörpert keine politische Filiation und kein patriarchales Totem. Führer Trump ist ,,a little bit different “: Familienmensch, aber nicht Stammhalter; Cheerleader (root on), nicht Stammeswurzel (rooted in); still und großzügig, aber von Großmannssucht (largess); Billionär und nicht elitär. „It's, um, amazing.“

Wie ist die Doppelgestalt auszudeuten, Trumps ferne Nähe, die von der Wähler*innenschaft hingenommen wurde? Zunächst eine These zum patriarchalen Muster: Trump vs. Clinton war kein ödipales Drama, in dem eine infantilisierte Bevölkerung dem Non-Du-Père folgen und die Mutter zurückweisen musste, um an der phallischen Macht des Vaters irgendwann zu partizipieren. In dieser billigen Psychoanalyse des Nationalen wäre die mimetische Rivalität zwischen Vater und Sohn wunsch- und wahlleitend: Der Wunsch, zu sein wie Mann/Vater Trump, erfüllt sich nur unter Akzeptanz des paternalen Verbots der Machtgleichheit. Die ödipale Sicht verankert die politische Beziehung von Wählerschaft und Souverän in der idealisierenden Neurose, dem double bind von Imitationsverbot und -gebot, und dem resultierenden Aufschub des Vater- bzw. Königsmords.

Die Ernennung Trumps setzt nicht das ein, was Lacan die ,Vaterfunktion“ nennt. Es ist eher die Einführung Amerikas in eine symbolische Unordnung, der Einsturz eines Zeichensystems. Die Umwertung aller American values rührt an allem: politischer Distinktion, Gewaltenteilung, Pressefreiheit, bis hin zur Unschuldsvermutung gegen Kinder, die 2018 an der mexikanischen Grenze in Käfige gesperrt wurden. Früh fiel das Inzesttabu: „If Ivanka weren’t my daughter, I'd be dating her", sagte Trump bereits vor den Wahlen. Für die puritanische USA ist es bemerkenswert, dass dieser Tabubruch (wenn schon nicht alle anderen) hingenommen wurde. Nach kurzer Verwunderung über die moralische Konturlosigkeit Trumpscher Männlichkeit finden wir bei Claude Lévi-Strauss Aufschluss über die Tragweite der Transgression: „Der Inzest ist eher sozial absurd als moralisch verurteilenswert. Das Inzestverbot ist weniger eine Regel, die es untersagt, die Mutter, Schwester oder Tochter zu heiraten, als vielmehr eine Regel, die dazu zwingt, die Mutter, Schwester oder Tochter anderen zu geben“ (zit. n. Girard 1999: 346, meine Hervorhebung).

„Sozial absurd“ ist das bessere Stichwort für diese Präsidentschaft, die ihr Basislager jenseits moralischer Urteile aufgeschlagen hat. Das Exogamiegebot ist bekannterweise nicht nur gemeinschafts- und kulturstiftend, sondern konstitutiv für die politische Funktion des Geschlechterunterschieds im patriarchalen Kapitalismus, wie von Gayle Rubin zu lernen ist. Die Frau wird zur Ware, durch deren Zirkulation Gesellschaft in Form von Tauschbeziehungen unter Männern organisiert wird. Männlichkeit entsteht dadurch, dass sie andere Körper, durch Objektivierung als weiblich signifiziert, tauscht. Trump behält demnach kulturvernichtend die Tochter für sich, und kann mit dem gesellschaftsbildenden 
Traffic-In-Women (Rubin 2011) so wenig anfangen, dass er auf Kosten eigener Mannhaftigkeit Ivanka zu seiner Beraterin und intima macht. Während er ein beispielloses trafficking von migrantischen Körpern initiiert, schließt Trump sein migrantisches trophy wife Melania im Schrank weg. Dieses Clan-Denken, das sexuelle und politische Beziehungen in der eigenen Familie knüpfen und behalten mag, ist konkret anti-sozial und anti-gesellschaftlich, denn es verweigert den konstitutiven Frauentausch. Mag er also ein Vater sein, so kann er niemals der VaterUrsprung der symbolischen Ordnung - sein.

Welche Figur innerhalb der amerikanischen Familie bzw. der clan-zentrierten Plutokratie der USA zeigt Trump an? In René Girards (1999) Lektüre von Bronislaw Malinowski (1962), der passenderweise nach der Gültigkeit des Ödipus-Komplexes in primitiven Gesellschaften fragt, findet sich ein Hinweis: Wie Girard schreibt, erfüllt bei den Melanesiern der Bruder der Mutter die symbolischen Funktionen des Vaters. Letzterer prägt zwar das direkte Lebensumfeld des Kindes, verkörpert aber nicht das , ,[...] Ideal im Freudschen Sinne des Ichideals oder des Über-Ich" (Girard 1999: 273). Die Auslagerung der Idealisierung auf den Onkel gibt der Konfliktbeziehung zur Autoritätsfigur freieren Raum, und ,,[...] die Spannungen zwischen Onkel und Neffe treten gerade deshalb so klar zutage, weil sie das Kind nicht in Widersprüche einschließen. [...] die Mimetik ist so kanalisiert, daß der Wunsch nicht sein eigenes Hindernis zum Objekt nehmen wird“ (ebd.: 273). Dies hat zwei Folgen: Zum einen hat der shitty racist uncle größeren Spielraum im Bereich der Despotie, der Tabuverletzung und der Aufstellung paradoxaler oder folgenloser Forderungen (,Build the Wall', ,Lock her up'), denn Onkel und Neffe/Nichte müssen nicht zusammen leben: ,,[...] die beiden Bereiche berühren sich nur an genau festgelegten Punkten, um zum gegebenen Zeitpunkt die Initiation des Nachahmers innerhalb der Kultur sicherzustellen“ (ebd.: 274, Hervorhebung im Original). Hier lässt sich die Politikferne durchschnittlicher Trump-Wähler* innen aus dem flyover country erahnen, für die Gesellschaft im übernächsten Dorf stattfindet. Der zweite Punkt ergibt sich aus der punktuellen Nähe: Die übergriffige Macht des Onkels lässt sich freizügiger genießen, denn die eigene Mischung aus Konkurrenz und Admiration, Imitation und Gegnerschaft, hält sicheren Abstand und muss sich nur sporadisch abgleichen. Distanzierte Idealisierung ist das Verhältnis zur ausgelagerten und damit entschärften Vaterfigur ,Onkel Trump‘.

Ein direktes Ergebnis dieser Onkelfunktion ist in der Unite the Right-Rally in Charlottesville 2017 ausgedrückt: Seit Trump kann die neue Rechte - von Roy Moore bis zur neofaschistischen Alt-Right - ihr Projekt weißer Dominanz ungehemmt und neurosenfrei in die amerikanische Geschichte und Gegenwart einschreiben. Der ödipale Aufschub revisionistischen und rassistischen Wollens ist nicht mehr nötig. Kein Vater muss gemordet werden, der die Regeln des Anstandes (den moderate republican racism) organisiert. Die Berührungspunkte der Alt-Right mit Trump sind bisher eben dies, punktuell, aber sie geben den rechten Protagonisten Gewissheit, auf dem Weg der Initiation richtig und geschützt zu sein: Der Präsident ist bester Kumpel und ferner Vater in einem. Die Anzeichen der Wahlverwandtschaft zwischen White Nationalists und Trump mehren sich mit jedem Tag. 
Auch dem Rest der Regierten bietet Onkel Donald Gelegenheit zur distanzierten Nähe. Man erträgt den Präsidenten wie den shitty racist uncle auf der Familienfeier. Seine Transgressionen lösen zugleich Scham und Schaulust, Kopfschütteln wie Kopfschmerzen aus: Trump, das abjekte Ideal. ${ }^{3}$ Erste Effekte des Systems Trump: Die neue Rechte dreht unneurotisch frei und erhält Spektakelcharakter; die politischen Gegner dagegen sind mit ihren Hemmungen alleingelassen - Nicht-Vater Trump vertritt nicht ansatzweise eine normative Ordnung, an der sich Opposition ödipal abarbeiten könnte.

Von den klassischen Beispielen Claudius (Hamlet) und Kreon (Antigone) abgeleitet, hat das popkulturelle Archiv der USA viele solcher Nicht-Vaterfiguren gespeichert: Der Präsident ist ein Amalgam des hochemotionalen Redneck-Tölpels Donald McDuck, seines raffgierigen Onkels Scrooge, sowie des ,bösen Onkels“ Charlie in Hitchcocks Shadow of a Doubt (1943), des skrupellosen Jason in Faulkners The Sound and the Fury (1929) oder Scar aus Disneys The Lion King (1994). In diesen Texten deutet sich eine zweite, unterschwellige Funktion des Onkels an - der Normenerhalt ex negativo: Onkel Donald lässt eine Reihe toxischer Männlichkeiten als völlig normal und moderat erscheinen. Sie erscheinen notwendig, denn sie stützen das freie Radikal Trump ab und scheinen somit einen Rest symbolischer Ordnung aufrecht zu halten. Wir erinnern, wer gemeint ist: Vizepräsident und professioneller Homophobiker Mike Pence, Ted , The World is on Fire ' Cruz, oder General James Mattis, verantwortlich für das sog. ,Mukaradeeb Massacre". Jede/r ultrakonservative Nutjob, und schließlich wir selbst, präsentiert sich als einer jener ,adults in the room“, die das namenlose Schreiben „I am part of the resistance in the White House" an die New York Times herbeizitierte (Anonym 2018). Mithin ist jede*r erwachsener als Donald J. Trump oder...

\section{An Evil Toddler and Besuited Chucky Doll}

Der Rückgriff auf die klassische Kulturanthropologie war etwas anstrengend, und mit einem zweiten Müdigkeitsanfall stellt sich die Frage, ob eine ,postbürgerliche Nation' (Heiner Müller) wie die USA sich für diese abgeschmackte Version bürgerlicher Tiefenpsychologie eignet, die nun unvermittelt von dem Barbar Trump de-rangiert worden wäre. Die Angriffe der Establishment-Demokraten lesen sich jedenfalls wie Zeitreisen in die bürgerliche ,Nationalfantasie ' der 1970er und 1980er Jahre, deren brüchigen Gefühlskonsens Lauren Berlant (1997) beschrieben hat. Nicht zufällig bedient die Strategie der Demokraten um das ,russian meddling' eine sentimentale Blockmoral des Kalten Krieges, in der Donald

\footnotetext{
${ }^{3}$ Mithin ein Grund für die derzeitige Schwierigkeit der Amerikaner*innen, passende Comedy zu machen: laut John Limon basiert amerikanische Stand-up-Comedy auf dem „standing up of abjection“ (Limon 2000: 4 f.). Dies ist erschwert zu leisten, wenn das Abjekte auf zwei Beinen im Weißen Haus herumstolziert.
} 
Trumps Beziehungen mit Russland als ,un-American activities' aufgerufen werden. Ein verdrehter McCarthyismus, der eine eher vergilbte Reinlichkeitssehnsucht der Supermacht USA beschwört. Im Vergleich ist die Alt-Right gegenwärtiger, wenn sie ,Trump+Putin` als postmodern-autokratische Männerromanze zugleich veralbert und wertschätzt.

Ist Trump ein temporal lapse, ein Zeitenbruch in eine andere Epoche, wäre diese anders zu bestimmen. Begegnungen mit dem Idioten stellen immer Zeitreisen - ,Make America Great Again" - oder Zusammenbrüche progressiver Zeitlichkeit dar. Die filmische Dystopie Idiocracy (2006) beleuchtete bereits vor Donny jenes Drehen an der nationalen Uhr, das eine Regentschaft der Dummheit bewirkt. Der Idiot öffnet das Fenster in eine ,einfachere 'Vergangenheit, wo Selbstjustiz noch hilft (z. B. Slingblade, 1997), ${ }^{4}$ oder in die technokratisch-entmenschte Zukunft (Charlie, 1978). Tatsächlich lädt Trump zum temporalen Kurzschluss ein: Ich selbst habe ihn voreilig als „walking talking 1930 s America“ (Strick 2016: 113) eingeordnet. Viele Kommentare teilen die Perspektivierung nach rückwärts und erfühlen die Trump-Epoche warnend als American Weimar (Liam Kennedy). Die Hoffnung ist, einer rituellen Wiederkehr demokratischer bzw. präfaschistischer Krisenmomente frühzeitig auf die Spur zu kommen. Die harte Rechte hat bereits länger ähnliche Reflexe und denunziert den vermeintlichen ,liberalen Konsens' als Wiederkehr eines radikalen cultural marxism der 1920er Jahre (vgl. Kimball 2007) und kopiert dabei selbst die antikommunistische Rhetorik der Nationalsozialisten.

Solche Rückschauen in Adoleszenzphasen kapitalistisch organisierter Demokratien, in Deutschland und anderswo, müssen Trump aber nicht als Durchgang verstehen, als eben rituelles Auftauchen einer hoffentlich kathartischen Krise der Demokratie. Vielleicht ist Trump eher das potenziell endlose Fegefeuer der Jugend; immerhin gibt es kein Indiz, dass nach Trumps populistischem Exzess etwas anderes folgt (sei es der ,reale' Faschismus oder die Rückkehr zum demokratischen ,Normalzustand'). Unter Bezug auf den Psychoanalytiker Mario Erdheim hat die Kulturwissenschaftlerin Gabriele Dietze angemerkt, dass in säkular-komplexen Gesellschaften wie den USA jene Initiationsrituale auf dem Rückzug sind, die als Schwellenerlebnisse das Erwachsen-Werden organisieren. Es droht Adoleszenz als endloser Schrecken: „Die Katastrophe der Pubertät findet keine geordnete Form mehr, die mit der Anerkennung eines Erwachsenenstatus abgeschlossen wird" (Dietze 2006: 42). Die Populärkultur, so Dietze, ersetze nun glücklicherweise jene religiösen Übergangsriten, in denen Angstlust und der Ekel am eigenen und fremden Körper eingezäunt und kulturalisierend überwunden werden. Anhand von Horror-Filmen wie Texas Chainsaw Massacre (1974) oder Child's Play (1988) argumentiert Dietze, dass das Genre des Splatterfilms dazu Szenen präödipaler

\footnotetext{
${ }^{4}$ In Slingblade (1997, Regie: Billy Bob Thornton) löst der geistig zurückgebliebene Protagonist ein soziales Problem neoliberaler Gegenwart, indem er das archaische Modell der Selbstjustiz für einen Moment wieder gangbar macht. Danach geht er, der am wenigsten Verstand, Status und Sozialität zu verlieren hat, zurück in den Zeitstillstand der Irrenanstalt. Diese Option haben wir nicht; Trump-Wähler*innen denken, sie hätten sie, wie wir später sehen werden.
} 
Prägung aufführt. Damit ermöglichen sie ein Durcharbeiten frühkindlicher Trennungsängste, die Konfrontation mit präödipalen Körper- und Egotraumata, an deren Ende die Festigung der Egoformation steht. Die Parallele sei hier weniger, dass wir gebannt einen ,Demokratie-Splatter" verfolgen, in dem Donald Trump als ,Leatherface‘ oder ,Chucky die Mörderpuppe ‘ auftaucht. Eine müßige Frage würde entstehen: Was arbeiten der Neoliberalismus, die USA oder wir selbst mit dieser Gruselshow durch? ,Bitte nicht den Horror-Clown', flehte die Hamburger Morgenpost zum Wahltag 2016.

Ohne Dietzes luzider Argumentation gerecht zu werden, bietet sich ein interessanterer Schluss an: Trumps infantiler Zugriff auf die Demokratie gleicht einem Durcharbeiten präödipaler Angstwelten. In Ermangelung von Vaterfunktionen und neurotischer Struktur tritt die frühkindliche Organisation der Donaldschen Psyche hervor. Anti-Ödipus Trump folgt genau dem Textbuch Melanie Kleins: Mutter als Lust- („Grab’em“) und Hassobjekt („Lock her up“), Penetrationsangst (,Build the Wall") und Penetrationslust (,these big beautiful hands“). Unerschöpflicher Körperhorror des Bakteriophobikers Trump, der sich 2016 nach seinem Interview mit FoxNews-Reporterin Megyn Kelly artikulierte: ,You could see there was blood coming out of her eyes, blood coming out of her wherever." The Horror, the horror.

So kommt das präödipale Kleinkind, der evil toddler, ins Weiße Haus. Georg Seeßlen hat bereits eine ähnlich infantilisierende Lesart angeboten, wenn er $\mathrm{Mr}$ Smith goes to Washington (1939) mit dem Einstieg Trumps verschaltet (Seeßlen 2017: 34 f.). Frank Capras Klassiker des demokratischen Populismus zeigt den Weg des amerikanischen Kindmannes ins Herz der Politik, gefasst als juvenile Reise an die eigenen Körpergrenzen. James Stewarts $M r$ Smith ist ein politisch naiver Bachelor und Boyscout; er agitiert mit Pfadfindertaktiken und Kinderarmeen gegen einen verkrusteten Machtapparat und die eigene Ohnmacht. Smiths Zeichen ist der stoische Arbeitseifer des Kindes: nach seinem sog. Filibuster - im Kongress liest Smith die gesamte Constitution vor, um ein wichtiges Votum aufzuschieben - bricht er erschöpft zusammen. Capras democratic drama ist wie der Horrorfilm ein Body Genre (Williams 1991), das viszeral durchlitten werden will: Erst mit dem Entgleiten der Körperkontrolle wird der American spirit geheilt. Smith entsteht wieder als populärer Volksheld und mannhafter Nationalkörper, dessen Opferbereitschaft im Kampf gegen das ,Monster Korruption“ den Systemglauben erneuert und zuletzt die Frau an seine Seite steuert. Über boyishness und Außenseitertum hinaus ergeben sich vielfache Parallelen: End the Graft (Capra) entspricht Drain the Swamp (Trump); wird Smiths naives Sprechen als boyscout-babble gezeiht, degradiert Trumps Diskurs sich selbst zum juvenilen „locker-room talk“.

Weitere Parallelen zwischen Trumps und Capras Populismus liegen nahe, kaschieren aber nicht die entscheidende Differenz: So sehr wir es ersehnen, Donny Boy ermüdet nicht, kein klimaktischer Kollaps leitet zur Reifung und damit Systemstabilisierung über. Da sich kein Schwellenerlebnis und relief einstellt, 
dauert die „Katastrophe der Jugend“ (Dietze 2006: 43) ewig. Das präsidiale Tun erschöpft sich u. a. deshalb nicht, weil es früh in ein paranoides double bind eigener Machart eingespannt wurde: Regent Trump beschwört bereits vor Amtseinführung die Gleichzeitigkeit von Gehindert-Werden (durch Opposition, Medien, Obama, den ,Deep State', die ,Rigged Election“) und Schon-Alles-ErreichtHaben: „Donald Trump is already helping the working class“, sagt sein Tweet vom 30. November 2016. Eine unmögliche Politik folgt, die nichts tun kann und alles erledigt hat. In dieser Kopräsenz von Sabotage und Sieg, die später noch wichtig werden wird, bleibt nur ein Weg: jener der Arbeit.

Seeßlen und Metz haben in ihrer rezenten Textsammlung zum Rechtspopulismus dessen Arbeitsethos herausgearbeitet. Der Rechtspopulist ,an sich“ pflege, so die Autoren, einen gedämpften Männlichkeitskult, denn er ,verkörpert nichts als Arbeit [...]. Er verspricht Arbeit durch seinen Körper und Hass auf alle NichtArbeit. Er sexualisiert diese Arbeit, lange bevor er das in seine Rhetorik einbaut, und sexualisiert noch mehr den Hass auf Nicht-Arbeit“" (Seeßlen/Metz 2018: 75). Trumps ,Regierungsarbeit' ist eingefasst in eine dauernde Ökonomie der größten Effizienz und Minimierung des Kraftverlustes; von der etwaigen Sexualisierung werde ich später sprechen. Erinnert sei daran, wie er im Golfmobil in Taormina (Sizilien) Energie einsparte, und die anderen sechs Staatsoberhäupter der G7 zu Fuß vom Photoshoot zum Konferenzort wandern ließ (Manchester 2017). Es gibt keine Spaziergänge in Trumps soziophober Werktätigkeit, keine Downtime, damit ein ewiger Handlungsimperativ aufrechterhalten wird und das Selfmade-Ego fortbesteht. Lügen ist ebenso Arbeit wie der Kampf gegen die Lügen; pausenloses Senden auf Twitter zu jeder Tages- und Nachtzeit; permanenter Wahlkampfmodus auf den so wichtigen Rallies; der Arbeitscharakter des Unterschreibens auf executive orders inszeniert in ständiger Wiederholung. Selbst der Dauerkonsum von FoxNews erscheint im präsidialen Tagesplaner als executive time, und Golf spielen in Mar-a-Lago ist noch Arbeit am ignoranten Selbst: „I'm working on my handicap.“

Wie in der oft gehörten Charakterisierung ist Trumps Arbeit also durchaus dem deal-making verwandt, denn dieses ist a priori unabschließbar. Es gibt immer den nächsten Deal und gemäß ihrer infiniten Struktur ist diese Arbeit zur Kunst erhoben: „Making money is art \& working is art \& good business is the best art Andy Warhol“", so twittert ,45`am 09.03.2015 unter Berufung auf den PopArt-Künstler. Einziger Gegner dieser immerwährend unabgeschlossenen Arbeit ist der beständig drohende Zerfall, das Abschlaffen und Nachlassen der Anspannung: ,low energy“ wie The Don einst dem Vorwahlgegner Jeb Bush attestierte. In der endlosen Abwehrarbeit zeigt sich der strukturelle Proto-Status des Dealmakers: nie völlig ausgeprägt, nie in der Welt angekommen, immer im Vorstadium der Egoabsicherung ackernd. Bei Klaus Theweleit, der eine wichtige Quelle für die ,Arbeits'-Hypothese von Seeßlen und Metz darstellt, findet sich die Bedeutungsmatrix der permanenten Tätigkeit jener, die abseits ödipaler Formeln des Erwachsenwerdens ihr Werk tun: Trump exemplifiziert einen jener ,,[...] nichtzu-Ende-Geborenen, die ihr Ich/Wir in ständiger Arbeit (Unterwerfungsarbeit) gegen den drohenden Zerfall erzeugen müssen“ (Theweleit 1978: 283). Die kanonischen Alltagsattribute der Persona Trump - immer gleiche Anzüge, Gesten und 
Slogans, Diet-Coke, Twitter-Kaskaden, Rallies, Unterschriften, FoxNews, überlanges Händeschütteln - werden auf dieser Folie als Erhaltungsmaßnahmen dessen lesbar, was Theweleit als ,Phantasma des Körperpanzers“ der faschistischen Männlichkeit zuordnet:

„Sich selbst Kommandos geben, sich Zusammenreißen, die ständige Beobachtung/das Körpertraining, [...] ,Männliche‘ Haltung, aufrechtes Wesen als Demonstration [...] und viele weitere ähnlich strukturierte Tätigkeiten können, soweit sie überwiegend der Erhaltung der Ich-Stabilität dienen, als die alltäglichen, die ,zivilen“ Formen des weißen Terrors verstanden werden“ (ebd.: 286 f.).

Verkürzt gesagt ist der Theweleitsche Faschist - wie der shitty racist uncle - damit von den hemmenden Dynamiken bürgerlicher Sexualpsychologie befreit: Seine Männlichkeit richtet sich gegen die gefährlichen Vermischungen heterosexuellen Begehrens, hebelt den Vater zugunsten der Waffenbrüder aus, die libidinöse Energie fließt ungebremst in die gewaltsamen Grenzziehungsarbeiten am einheitlichen, unverbrüchlichen und überzeitlichen Volkskörper, oder eben Trump-Tower. ${ }^{5}$ Aus der Abwesenheit ödipaler Verbindlichkeiten ,[...] resultiert das für den Faschismus typische Verhältnis des Wunsches zur Politik: sie wird direkt besetzt, ohne Umwege, ohne Mama/Papa-Stempelungen, ohne Codierungen durch Konventionen, Institutionen oder historische Bedingungen“ (ebd.: 280 f.). Das ist treffend formuliert: Kein Ideal und kein Erschöpfungshorizont regeln den Übergriff des nicht-zu-Ende-Geborenen auf die demokratische Ordnung. Nicht Genealogie, noch Takt, Zeitgefühl, Neurose (Aufschub), Reflexion oder Schlaf bremsen Handlungszwang und egomane Arbeitswut des Donald Trump, oder...

\section{A Badly Drawn Fascist}

Erliegen wir nicht der vereinfachenden Facette dieses temporal lapse: Die Invektive und Analyse erringt nicht allein die Erkenntnis, dass Theweleits faschistisches Körper- und Selbstzüchtungsprogramm nun in der Gestalt eines neoliberal-selbstorganisierten Tycoons mit Zersetzungspanik und tyrannischen Tendenzen wiederkehrt (McNeill 2016); Kulturkritiker Henry Giroux hat dafür übrigens den Begriff der „,neoliberal fascist politics“ (Giroux 2018) in den Ring geworfen. Immerhin aber ist ein Faschist deshalb bedrohlich, weil er die paranoide Ich-Stabilisierung zum rassistischen Gesellschaftssystem und totaler Mobilisierung ausbaut, um mit extremer Effizienz eine global-denkende Nekropolitik zur Todesproduktion für alles ,Andere“ voranzutreiben. Es sei an die bislang relative

\footnotetext{
${ }^{5}$ „Noch immer schaffen die Menschen an einem Turmbau von unermeßlicher Höhe, zu dem sie ein Geschlecht, einen Zustand ihres Seins mit Blut, Qual und Sehnsucht auf den anderen schichten. Langsam, unendlich langsam wachsen seine Quadern der Gottheit entgegen“" (aus Ernst Jüngers Kampf als Inneres Erlebnis, zit. n. Theweleit 1978: 68). Kürzer fasst sich abermals Sarah Palin: „He builds things that touch the sky“ (CBSN 2016).
} 
Ineffizienz Donaldscher Regentschaft erinnert, die zwar gerne damit kokettiert, potenziell an einige Hebel des Totalitarismus heranzukommen („I have the absolute right to PARDON myself", oder der seit Dezember 2018 angedrohte Ausnahmezustand zur Finanzierung der Mauer). Auf der anderen Seite regiert The Don durch Vernachlässigung des Politischen selbst. Avital Ronell erwähnt diesen

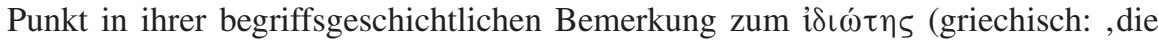
private Person $\left.{ }^{\prime}\right):,[\ldots]$ the Greek approximation or anticipation of stupidity would have to be located in the prepolitical, in the forgetting of politics" (Ronell 2002: 41, meine Hervorhebung).

Der griechische ,idiotès ' ist dadurch privat und eben Idiot, dass er vergisst, hinaus zur Agora zu gehen und am Meinungsbildungsprozess teilzunehmen. Das oben zitierte anonyme Schreiben an die NYT liefert dazu die Anekdote, dass Trumps Entscheidungspolitik beeinflusst werden kann, indem man Papiere vom präsidialen Schreibtisch entfernt. Trump regiert und irritiert nicht durch/ als Geschichtsrevisionismus oder Backlash, sondern als Vergessenheit: was die Agenda ist, was er letzte Woche gesagt hat, wer seine Getreuen sind, was er mit Hillary vorhatte, was NATO ist, was Demokratie, was Totalitarismus. Wichtig ist ihm Politik nur an der Grenze von Ego und Öffentlichkeit: Es zählt das daily drama des Tweets, der Moment der Gegnerbeleidigung, die instantane Bildlichkeit des Unterschrieben-Habens, die Impromptu-Emotion der Rally, die rituelle Beschwörung der Mauer, der Sabotage, des witch hunt. Die Restitution des, weißen Terrors' (Theweleit) nimmt die Form eines egomanen Maelstroms des Vergessens oder - anders gefasst - eines totalen presentism an.

Das bedeutet, dass Trump keine ,Retraditionalisierung ' vorlegt, die dem transnationalen Rechtsruck gerne zugeschrieben wird, als sei er Umkehr und Rückkehr des Überkommenen bzw. Verdrängten. In der Echtzeit-Ästhetik Trumpscher Regierung interessiert Tradition - z. B. republicanism - nur als direkter Gebrauchswert und pragmatischer Diskursbeschleuniger. Tradition ist nur Genre-Zeichen und momentane Besetzung einer Form. Nicht umsonst ist die kürzeste Jeremiade aller Zeiten - MAGA - völlig inhaltsleer, sowohl ,again ‘ als auch ,great` spezifizieren nichts. Betreibt Trump die „Ästhetisierung des Politischen" (Walter Benjamin), die den Faschismus kennzeichnet, so bedient sich diese weniger bei der tief reichenden Geschichte und Stilistik weißer Rassennobilität, als vielmehr bei den präsentischen Käfigformeln, ad-hoc-Dramen und Mikrofaschismen des Reality TV. Misha Kavka hat unlängst diese Linie herausgehoben und das Realitätsfernsehen als Hauptlogik Trumpscher Performance bestimmt: „Im Zentrum dieser Formel [...] sitzt Trump als Manifestation eines irrationalen, vom Affekt getragenen Gesetzes, das ihn als [...] , obszöne Figur des Vergnügens ‘ markiert [...], einem wissenden Vergnügen an Pathos, Zurschaustellung und übertriebener Performance" (Kavka 2018: 159), so beschreibt er konzise die voyeuristische Spektakel- und Fremdschäm-Logik des Genres.

Über Kavkas wichtigen Befund hinaus lohnt sich die Historisierung dieser Reality-Formel. Der Journalist Kurt Andersen hat zuerst auf den ästhetisch-performativen Kanon des Wrestling verwiesen, der vor dem Realitätsfernsehen die Phänomenologie von Spiel und Ernst, Stillosigkeit und Spektakel, geskriptetem 
Drama und authentischer Dämlichkeit auszumessen begann. Das Wrestling biete die Explikationsfolie für Trumpsche Überzeugungskraft bei gleichzeitigem schlechtem Stil:

\footnotetext{
„Wrestling, WWE, is, if not the key, a large key, to the Donald Trump phenomenon as we're experiencing it today. What they started doing in the '80s more than they've ever done before when he got involved in world wrestling, is this blurring of the lines between the characters they're playing and ,I'm pretending to be angry at you, Hulk Hogan,' and bringing that outside the ring and making it kind of real and blurring for everybody what was real and what isn't" (Johnson 2016).
}

Andersen rekurriert hier auf die spezifische Vermischung von Show und Wirklichkeit, die im Wrestling-Jargon mit dem Begriff ,kayfabe‘ ausgedrückt wird. Der Begriff bezeichnet jene Illusion des ,echten“ Dramas und Sports, die das durchgeskriptete und formelhafte Format des Wrestling unter allen Umständen aufrecht erhält. Mit Trump ist ,kayfabe“ vom Männercatchen via The Apprentice in die Globalpolitik eingewandert, und wird von vielen verkürzend als ,postfaktische Ära' interpretiert. Diese Migration von Genreregeln ist äußerst folgenreich, wie die Theaterwissenschaftlerin Sharon Mazer in ihrem wichtigen Artikel „Donald Trump Shoots the Match“ (2018) herausarbeitet, und bringt einen weiteren Punkt für die Erörterung Trumpscher Männlichkeit ein: Das Wrestling liebt besonders den Gilb und Schmalz der partizipierenden Männlichkeiten - ihre relative Überkommenheit ist entscheidender Teil des Sehvergnügens. Machoman Randy Savage, The Undertaker, The Bush Wackers, Hulk Hogan, Rick Flair heißen die Helden des Genres, dem Trump bereits seit den 1980er Jahren nahesteht. Solcherlei Schablonenfiguren karikieren diverseste Männlichkeiten, stilisieren deren Exzentrik, Americanness, regionale oder ethnische Identität ins Dämlich-Anrührige.

Im „Battle of the Billionaires“ (WrestleMania 23, 2007), schickte Trump den afroamerikanischen Wrestler ,Bobby Lashley“ (eine Art Booker T. Washington-Figur) gegen Sportimpresario Vince MacMahon und seinen samoanischen Kämpfer ,Umaga' (die wilde Bestie) in die Arena. In solchen Szenarien agiert das Wrestling soziale Konflikte aus, und macht die alten Race- und Class-Relations der USA auf volkstümlich-ironische Art erfahrbar: weiße Mogule schicken ethnisch-markierte Fantasiekörper in den Kampf um Supremacy, und scheuen sich nicht, im opportunen Moment dem Gegner einen Klappstuhl über den Kopf zu ziehen. Die Showmanship des Wrestling war ein natürliches Areal für die walking brand Trump, der zwischen theatralisch brüllenden Kontrahenten mit stereotyper NY-Coolness und kontrollierter Stimme agierte. Sein Gladiator gewann übrigens das Match, und MacMahon wurden im als ,Hair vs. Hair'-gebrandeten Event die Haupthaare abrasiert.

Trump und seine Persona surfen bewusst auf diesem ebenso selbstironischen wie corny Genre, das dem von Richard Dyer beschriebenen ,Peplum“ nahesteht (1997) und den Handlungsmöglichkeiten, die es bereit stellt: Große Melodramen und Showdowns, Erniedrigung des Gegners, schmutzige Tricks, Lügen, Bluster und Boasting, durchexerziert von stillos zurechtgesägten Muskel- und 
Manager-Männlichkeiten. Der faschistische Körperpanzer begegnet uns im Wrestling als öliges Clown-Monster und schlecht schablonierte Parade der Übermenschen-Diversität. Die Beleidigungsformel badly drawn fascist trifft dort, wo sie die ,schlampige Modalität‘ von Trumps faschistoider Männlichkeit beschreibt: dieser Führer ist tacky, boden- und geschichtslos, mit links gemalt, lächerlich, eine schmierige Karikatur in einem wrestling match. Attribuierungen wie ,geschmacklos', ,übergriffig', , White Trash', , sexistisch', ,rassistisch', , verlogen', ,zu dumm zum lesen' und ,quasi totalitär" ${ }^{\prime 6}$ bezeichnen demnach keine Mängel. Sie sind der Stil des Wrestlers/Politikers, seine Marke, sein Narrativ, das affektive Plus, the joy of it. Maßgeblich an Trump ist nicht eine jähe Wiederkunft des Präfaschismus, sondern dessen marktorientierte Vulgarisierung und Verkitschung als Dauerentertainment. Donnyboy sei der Herrenmensch von der leicht konsumierbaren Ungestalt, oder...

\section{The Political Equivalent of One of those Mutant Factory Farm Chickens with Breasts so Big It Can't Walk}

Noch erschöpfter sind wir einen Schritt weiter, wenn wir Trump Fascism nicht allein als Übergangsphase zu einem systemischen Totalitarismus, sondern als Daseins- und Regierungsform im Dauerpräsentationsmodus à la Wrestling verstehen. Dort treiben alle Aktionen ein simples und unabschließbares Narrativ (z. B. good vs. evil, MAGA) weiter, übertreiben es, bauen Konfliktpunkte und Spannungen auf und ab. Wie im Dealmaking (s. o.) wird die Realitäts- und Arbeitsebene der Show - das ,kayfabe " - niemals verlassen, sondern im Gegenteil deren Geltungsbereich stetig ausgeweitet. Mithin kann das ,System Trump“ so begriffen werden, wie man sich einer Aktion von Christoph Schlingensief nähert als rücksichtsloser Übergriff einer performativen Binnenlogik auf die übrige Welt, und deren nachfolgende Transformation. Das Leitthema zu Wahlkampf und bisheriger Regentschaft - ,admit nothing, deny everything, launch counterattack“ hätte zum verstorbenen Film- und Aktionskünstler gepasst. Es stammt von Trumps Ex-Strategieberater und Informationskrieger Roger Stone (vgl. McKew 2018).

Die Eigenmechanik greift über die Persona Trump hinaus, die wir bisher beleuchtet haben. Die Trumpsche Mutation von Demokratie erhält partielle Allgemeingültigkeit, wirkt wirklichkeitskonstituierend und systembildend. Als rezentes Beispiel steht das Supreme Court Hearing zur Personalie des Richters Brett Kavanaugh im Oktober 2018 zur Verfügung, dessen Berufung an den obersten Gerichtshof der USA für viele einen Systembruch darstellt. Wie schon im Ernennungsverfahren des konservativen Richters Clarence Thomas im Jahr 1991 (vgl. Dietze 2014: 357 ff.) fokussierte das Hearing auf Vorwürfe sexuellen Missbrauchs: Kavanaugh wurde beschuldigt, im Alter von 17 Jahren versuchte

\footnotetext{
${ }^{6}$ Henry A. Giroux schlägt den Begriff ,civil illiteracy“ vor (Giroux 2018), der die letzten beiden Punkte - undemokratisch und leseunfähig - zusammenfasst.
} 
Vergewaltigung und sexuelle Nötigung an der damals fünfzehnjährigen Mitstudentin Christine Blaisey Ford begangen zu haben; zwei weitere Frauen brachten ähnliche Vorwürfe vor. Die Psychologieprofessorin Ford sagte aus, während einer Party habe der stark alkoholisierte Kavanaugh sie in einem Zimmer festgehalten und gewaltsam zum Geschlechtsverkehr zu zwingen versucht. Ein Fraternity-Kollege habe amüsiert zugeschaut. In einer kurzen FBI-Untersuchung wurden keine ,Beweise ' für den Tatbestand gefunden (bekanntes Problem bei Vergewaltigungen), dafür zahlreiche Hinweise auf die exzessive frat-boy-Vergangenheit des Kandidaten. Kavanaugh wurde vom republikanisch dominierten Senat mit $50 \mathrm{zu} 48$ Stimmen gewählt.

Die dramatische Logik des Wrestling griff auf das Verfahren über, als Kavanaugh am 27. September sein Eröffnungsstatement lieferte. Zwei Strategien wären normal und erwartbar gewesen: der Nominierte würde seine Statur als integrer Verteidiger des Rechtsstaats festigen, und/oder die Vorwürfe mit einem süffisanten ,boys will be boys' bagatellisieren. Entgegen der Konvention gab der Richter eine tränen- und zornreiche Performance verwundeter Alpha-Männlichkeit und beleidigter weißer Privilegierung zum Besten, bis zum Punkt der parteipolitischen Entgleisung: „This hearing is a national disgrace. [...] Since my nomination in July, there has been a frenzy on the left to come up with something, anything to block my confirmation." Eckpunkte der Rede waren: er trinke gerne Bier; er gehöre seit jeher zur rechtmäßigen Elite seiner Universität Yale; jegliche Anschuldigung sei ein dezidiert politischer Angriff der radikalen Linken auf seine Ehre als Mann, Yale-Absolvent und Republikaner, sowie auf den Rechtsstaat per se. "Search and destroy“, betitelte er die demokratische Strategie. Der republikanische Senator Lindsey Graham sekundierte im übertriebenen Ton einer Wrestling Feud, die ein extensives Zitat lohnt:

Graham: „This is the most unethical sham since I've been in politics and if you [Democrats; S.S.] really wanted to know the truth, you sure as hell wouldn't have done what you've done to this guy. Are you a gang rapist?"

Kavanaugh: ,No.“

Graham: „I cannot imagine what you and your family have gone through. Boy, you [Democrats; S.S.] all want power. God, I hope you never get it. I hope the American people can see through this sham. [...] God, I hate to say it because these [points to Democrats] have been my friends, but let me tell you, when it comes to this, you're looking for a fair process, you came to the wrong town at the wrong time, my friend. Do you consider this a job interview? [...] Would you say you've been through hell?"“

Kavanaugh: „I've been through hell and then some.“

Graham: "This is not a job interview.“

Kavanaugh: „Yeah.“ 
Graham: „This is hell. This is going to destroy the ability of good people to come forward because of this crap. Your high school yearbook. You have interacted with professional women all your life, not one accusation. You're supposed to be Bill Cosby when you're a junior and senior in high school. And all of a sudden you got over it. It's been my understanding that if you drug women and rape them for two years in high school, you probably don't stop" (zit. n. Roth 2018).

Grahams Dramaturgie folgt hier einem klassischen Wrestling-Szenario, in dem ein ,guter' Kämpfer (im Wrestling-Jargon das sog. Face) einem anderen beisteht, der durch den Villain (Heel) der Show beleidigt, hintergangen und um einen wichtigen Sieg gebracht wurde. Verweise auf die Skrupellosigkeit des Bösewichts und der Druck auf die Tränendrüse des Ehrgemordeten gehören ebenso zur Formel wie Appelle an das nationale Publikum, den Trick (Sham) zu durchschauen. In der Regel bereitet ein solcher Dramablock ein sog. Tag-Team-Match vor - zwei ehrgleiche Muskelbrüder vereinen sich gegen den Villain und seinen Schergen - mit dem Ziel, dass die moralische Welt zeitweise gerade gerückt wird, der Titel dem rechtmäßig ,Besten“ zufällt, und zugleich genug Drama anfällt, um das nächste $W W E$-Event zu motivieren. Ähnliches gilt hier: obwohl Kavanaugh - angesichts der derzeitigen Mehrheiten keineswegs überraschend - als Richter bestätigt wurde, firmierte das gesamte Verfahren als Totalangriff auf eine patriotische Männerelite, die durch harte Arbeit und Aufrichtigkeit an ihre Privilegien gekommen sei. Wrestling-Aficionado Trump kommentierte das abgeschlossene Verfahren mit warnenden Worten: „It's a very scary time for young men in America when you can be guilty of something you may not be guilty of."

Neben dem offensichtlichen Bruch mit den sakrosankten Prozessnormen einer Richterernennung finden wir hier die Ausweitung des paranoiden Systems von gleichzeitigem Sieg und Sabotage, das oben beschrieben wurde. Die Massierung ungezähmt-emotionaler, gekränkter und ,unterdrückter" Männlichkeiten - die gleichzeitigt gewinnen - ist deutliches Zeichen der kulturellen und politischen Zeitenwende. Der politische Prozess ist in die abgeschmackte Welt des Männercatchens überführt: Über das einzelne Match hinaus dauert das ,aggrieved entitlement" (vgl. Kimmel 2017: Kap. 1) dieser Herren an, niemals folgt Entspannung, die ideologischen Gräben werden stetig vertieft, neue Fehden werden vorbereitet. Hier tritt kein Patriarchat auf, das sich kühl gegen z. B. marginalisierte und emotional argumentierende Frauen verteidigt. Der modus operandi ist die emotionale Mobilisierung eines sich dauerhaft verletzt-fühlenden Bundes von frat-boys und privilegierten Reaktionären an den Schalthebeln der Macht, sowie deren jubelnden Fans.

In einer Welt jenseits des Wrestling wären simple Fragen entstanden: Wenn schon nicht Vergewaltigung für das Amt disqualifiziert, dann vielleicht kindische Insistenz auf das eigene entitlement, oder Ignoranz für die überparteiliche Aufgabe des Amtes? Nichts verfängt im Skript des Ehrenkampfes: ein offenkundiger politischer Sieg wird als Tiefenbeleidigung und Beweis für die Verkommenheit des Gegners verkauft. Der parteipolitische Fundamentalismus eines obersten Richters wird angesichts der erfolglosen Sabotage zur Selbstverteidigung und persönlichen 
Qualität. Emotionale Entgleisung ist Ausweis politischer Legitimation. Tatsächlich ist es weiten Teilen der USA derzeit egal, ob irgendjemand die oben genannten Nicht-Qualifikationen erfüllt. Wenn eine Verletzung durch den ultimate villain ,Feminismus, Political Correctness, Linke, Globalists, usw. ${ }^{7}$ schmierenkomödiantisch nachgewiesen werden kann, ist man der richtige Mann für's politische Amt.

Zentrale Strategie aller Arbeit im ,Prinzip Trump“ ist es demnach, das ,kayfabe' nicht zu unterbrechen. Jeder Kampf und jeder Sieg muss den nächsten Revenge-Plotpoint vorbereiten. Wie im Wrestling geht es weniger darum, Ereignisse und Realitäten geschickt für die eigene Narration zu instrumentalisieren. Diese republikanische Show produziert Ereignisse, und deren Instrumentalisierung geschieht, bevor sie stattfinden. ${ }^{8}$ Der Begriff einer ,Fiktionalisierung des Realen greift zu kurz um den fundamentalen Austausch politischer Rationalitäten und Realitäten gegen den partisan surrealism und die scripted reality der Republikanischen Partei zu beschreiben; die aktive Vernichtung konsensfähiger Realität ist Ziel des Systems ,Donald Trump“, or ...

\section{A Hairpiece Come to Life}

Wenn das Abgeschmackte der weißen USA zum Leben erwacht und auf die restliche Realität übergreift, geht auch ein Grundprinzip differenzsensitiver Kulturwissenschaft verloren: jenes der sog. , default position of whiteness ', das u. a. die Feministin Ruth Frankenberg (1993) beschrieben hat. Aus dem intersektionalen Feminismus heraus entwickelt, beschreibt dieses Theorem, dass innerhalb einer mehrheitsweißen Gesellschaft wie den USA die racial majority sich selbst nicht als ethnisch verfasst wahrnimmt. Sie versteht sich als unmarkierte Norm außerhalb von racial difference, von deren Unsichtbarkeit alle ethnischen Körper als sichtbar , andere" unterschieden sind. Illustrativ für die default-Position ist eine unterhaltsame Liste, die die afroamerikanische Nachrichtenplattform theroot.com vor kurzem herausgegeben hat. In seinem Artikel „40 Ways White People say ,White People“ without actually saying ,White People“" zählt Damon Young (2018) neurotische Verschiebungen auf, mit denen der weiße Mehrheitsdiskurs seine Teilhabe am systemischen Rassismus der USA verklärt und sich zur unmarkierten Norm mystifiziert: „Real Americans. Middle America. Working-class Americans. Patriots. Southerners. Christians. Evangelicals [...]“ (Young 2018).

In Erinnerung an Brett Kavanaughs Statement lassen sich weitere Einträge ergänzen: „top of my class at Yale; captain of the varsity football team; I like beer" waren sämtlich deutliche Hinweise auf Milieu, Habitus, Distinktion und racial belonging des Kandidaten. „It shrieks whiteness“, wie Richard Dyer zu Trumps ewig-blondem Haartoupet anmerkt (2018). Lindsey Grahams oben

\footnotetext{
${ }^{7}$ Fiktionale Gruppierungen, daher in toto in Anführung.

${ }^{8}$ Die Realitätskonstruktion und Produktion von instrumentalisierbaren Ereignissen ist auch in den Online-Aktionen der Alt-Right zu beobachten (vgl. Strick 2018).
} 
zitierter Verweis auf den afroamerikanischen Fernsehstar Bill Cosby, der kurz vorher wegen zahlloser sexueller Delikte verurteilt worden war, tat ein Übriges, um die Unterscheidung zwischen ,Yale student having fun " und ,serial rapist" mit rassistischer Deutlichkeit als color line zu bestimmen. Wir haben wiederholt dem Verlust der neurotischen Struktur nachgespürt, und finden ihn hier noch einmal bestätigt: die verwundete weiße Männlichkeit Kavanaughs muss sich im ,System Trump“ nicht hinter Allgemeinplätzen verstecken, sondern kann ihre whiteness als Anständigkeit, Schuldfreiheit und Anspruch auf Macht - in aller Offenheit und gegen ihre Angreifer einklagen. Die explizite Selbstpositionierung weißer Männer als systemisch bedrohte racial group, bislang den Vertretern des white nationalism vorbehalten, wurde vom Duett Kavanaugh-Graham selbstbewusst und gut sichtbar für die amerikanische Öffentlichkeit diskursiv vorgetanzt.

Lauren Berlant wies letztes Jahr auf den Verlust von whiteness als unsichtbarer Positionierung hin, als Identitätskategorie, die nur metonymisch verschoben artikulierbar ist. Seit der Inthronisierung des ,Big Man“ sei die machtvolle Identitätsneurose der weißen Mehrheitsgesellschaft einer Fleischwerdung des Unsichtbaren gewichen:

„It used to be possible to teach whiteness as ,unmarked “ - but if whiteness ever existed
that way outside of the white mirror, it's gone now. This rebooted electorate wants its whi-
teness enfleshed. Because it does not think of structural inequality as anti-American, it
thinks homeopathically that breaking the liberal difference/tolerance machine will stanch
loss, not engender in its name surprising devastations“ (Berlant $2017 \mathrm{a}$, meine Hervor-
hebung).

Mit whiteness als offen diskutierbarer Identitätsmarkierung schließen sich also weite Teile der konservativen Öffentlichkeit an jene ehemals emanzipatorisch gemeinte, ,linke' Apparatur der identity politics an, ohne ihre Fundamentalkritik an dieser aufzugeben. Ein weißer Trump-Wähler im Radiointerview auf NPR macht diesen performativen Widerspruch vor: „The blacks have their schools, so why can't we have ours? It's about time." Sieg und Sabotage kehren hier wieder und legitimieren die weiße Dauerkränkung bei gleichzeitigem Machtzuwachs.

In Verkennung historischer und gegenwärtiger Mehrheits- und Machtverhältnisse werden so white identity politics in Anschlag gebracht, die in erster Linie emotional greifen und eine gefühlte Deprivilegierung performativ gangbar machen - nicht nur bei der white working class, sondern, wie zu sehen war, bis hinauf zur juristischen Elite. Diese showhafte Identitätspolitik und die handlungsleitende Emotion des „aggrieved entitlement“ (Kimmel 2017) sind nicht mit emanzipatorischen Hoffnungen verknüpft. Die white identity politics z. B. eines Brett Kavanaugh investieren nicht in Ideale sozialer Gerechtigkeit; sie versprechen den ,homöopathischen“ Aufschub der Erosion weißer Normalität, ${ }^{9}$

\footnotetext{
${ }^{9}$ Wäre die Ermüdung nicht schon so weit fortgeschritten, könnten wir zwei Ausgangspunkten dieser Erosion nachgehen: im Fall von Kavanaugh die tatsächlich gewollte Dekonstruktion weißer Männereliten; im Fall der meisten Trump-Wähler*innen die kulturell, ökonomisch und
} 
deren Ästhetik mithin in wenig mehr besteht - Bier, Yale, varsity football - als in anachronistischem white kitsch. ${ }^{10}$ Die zahlreichen Teilhaber*innen am ,System Trump' hängen einem verblichenen und verlorenen Bild unhinterfragter weißer Privilegierung nach.

Dieses soll allerdings nicht restauriert werden: im Gegensatz zur klassischen Identitätspolitik, die auf Systemwandel, Repräsentationsteilhabe und empowerment von Minderheiten abzielt, geht es diesen white Identity Politics allein um eine Vermehrung der Stellen, an denen sich Mehrheiten verletzt, marginalisiert und unterdrückt fühlen können. In dieser Richtung ist auch Trumps realpolitisches Wirken zu verstehen: seine Maßnahmen schädigen vor allem das eigene Wahlvolk (z. B. Krankenversicherung, Einschränkung von Wahlberechtigungen) und schlagen aus dieser Prekarisierung populistischen Profit. Der Historiker Timothy Snyder (2018) schlägt für diese Logik den Begriff des sadopopulism vor: populistische Regierungen schädigen ihre Wähler*innenschaft gezielt, um diese Verletzungen anzuerkennen und eine kathartische Wende $\mathrm{zu}$ versprechen, die in wenig mehr besteht als dass der politische Gegner mehr Schmerzen erleiden werde als man selbst. Die sadopopulistische Wähler*in , can believe that he or she has chosen who administers their pain, and can fantasize that this leader will hurt enemies even more“" (zit. n. O’Toole 2018).

Das ,System Trump“ ist in dieser Beschreibung eine gewollte Eskalation, ein Aktionismus gegen gefühlte Verluste, die nicht behoben, sondern affirmiert, vervielfacht und aushaltbar gemacht werden sollen. Richard Dyer (2018) hat für diese Strategie eine naheliegende Metapher in Donald Trumps Haarpracht gefunden. Ich möchte seine Haaranalyse zu diesem Schluss fortführen: Trump hat dieselbe Funktion für seine Wähler*innenschaft, die das leuchtend-blonde Toupet für ihn selbst hat. Wie alle abgeschmackten Show-Techniken - vgl. Wrestling und racial kitsch - funktioniert ein Haarersatz nicht wirklich ersetzend: „comb-overs, toupees and implants [...] never really work", wie Dyer schreibt (2018). Auch Udo Walz merkt an, dass Versuche, Resthaar zu drapieren oder falsches Blond aufzusetzen, nur das Verlorene betonen: ,Je mehr man etwas verstecken möchte, umso mehr macht man darauf aufmerksam“ (Walz 2018).

Sie sind aber besser als nichts, und versprechen vage die Illusion einer restituierten Normalität, von der man weiß, dass sie immer bereits verloren ist. Erst das Toupet eskaliert die Glatze zum Mangel, und seine Binnenlogik zeitigt zwei Effekte: zum einen ist man permanent mit der eigenen Stilisierung beschäftigt,

\footnotetext{
sozial destruktiven Effekte des Neoliberalismus. Es sind also durchaus verschiedene Bedürfnisse am Werk, was jedoch nicht verhindert, dass weiße Elite und weißes Prekariat gefühlt dasselbe meinen: die für mich immer noch seltsam klingende Angriffskombination gegen ,the Left' und ,Globalists', gegen ,Feminists‘ und ,Corporate Elites' wird hier erklärlicher. Ebenso erhellt sich der performative Widerspruch, warum gegen vom Kapital organisierten ökonomischen Abstieg breiter Schichten ausgerechnet noch mehr Kapitalismus helfen sollte.

${ }^{10}$ In diesem Licht ist auch Melania Trumps kürzlich präsentierter ,Kolonialhelm‘ zu lesen. Vgl. zu racial kitsch auch Nyong'o (2002).
} 
arbeitet also heldenhaft am demolierten und geschwundenen Selbstbild (vgl. Berlant 2017b). Der Aktionismus des Verbergens macht die Einsicht und Affirmation der eigenen Prekarität, des ökonomischen, politischen oder frisurtechnischen Verlusts, erträglich. Zum anderen befeuert das Toupet den ,Mut der Verzweiflung dem Publikum gegenüber: Legitimiert von der eigenen Heldenarbeit am Verlust, können die belustigten und/oder mitleidigen Zuschauer*innen der erfolglosen Haararbeit - corny, tacky, fake - als Feinde ausgegrenzt werden. Nach dem Motto: ,wenn euch das nicht reicht, dann reicht euch nichts.' Aus dieser Aushandlung erhält man als Dividende den Stolz, authentisch trash, fake und white zu sein. Legitimation genug, die eigene Realitätsarbeit radikal über die der Anderen zu stellen - Trumps gelebter Kitsch schert sich schlichtweg nicht um jene Ideologiekritik, die wir gegen ihn vorbringen.

Toupet Trump ist eine Eskalation der Prekarisierung, die sich wie Schadensbegrenzung anfühlt: Man leistet sich den Horror des Onkels, die Peinlichkeit des Haarteils, die Weinerlichkeit des white kitsch, das Zerbrechen demokratischer Prozesse, und hofft, diese würden irgendwie irgendetwas Schlimmeres (z. B. ,feminist totalitarianism', ,cultural marxism') verhindern. Das ist das bargaining der Republikaner und der Trump-Wähler*innen: Getrieben von einer Nostalgie, von der man weiß, dass sie niemals eingelöst wird, hängt man sich im Verlorenen auf und verteidigt den eigenen Schmerz aggressiv. Die Aufkündigung gesellschaftlich teilbarer Gegenwart wird in Kauf genommen. Der Haarausfall avanciert zum Prinzip der Realpolitik; das Toupet herrscht über die bare Glatze, so wie Donald Trump herrscht über...

\section{The Barron Land}

„I'm so tired of being cynical.“ (Nabokov 1970: 111)

Dieser Aufsatz folgte Hinweisen aus der Trumpschen Dauerperformance, um den unterliegenden Mechaniken dieser Präsidentschaft und Auswirkungen auf die politische und kulturelle Landschaft der USA nachzuspüren. Es ist auch ein Versuch, Trump dahin gehend zu theoretisieren, dass er vom überaus lautstarken Symptom (z. B. einer Krise des Demokratischen) zum Ereignis, Akteur oder System transformiert ist. Damit will ich Ansätzen entgegentreten, die Trump als Aufregungspunkt nutzen, um mit der erwirtschafteten Empörungsenergie politischen oder erkenntnistheoretischen Druck auf etwas Anderes zu erzeugen; z. B. die derzeit wohlfeile Theorie, an der populären Obszönität des Präsidenten (und anderer Rechter) seien die Fehler linksliberaler Anständigkeits- und Gerechtigkeitspolitik abzulesen, zu der nun (z. B. populistische) Alternativen gefunden werden müssten. Solche Perspektiven betreiben unlautere Kraftumwandlung, denn sie organisieren aus der Anstößigkeit eines scheinbaren Symptoms Kritik an der scheinbaren Systemkrankheit. So mutiert die kritische Öffentlichkeit selbst zum Symptom des ,Systems Trump " und seiner stichwortgebenden Weltverzerrung, ein beunruhigender Prozess, mit dem wir sicher noch einige Zeit zu tun haben werden. 
Wir sind aber keine Ärzt*innen oder Symptomatolog*innen. Trump sollte nicht als Kuckuck perspektiviert werden, der lediglich anzeigt, wie spät es ist, sondern als sichtbarstes Untier auf einem Uhrwerk, einer arbeitenden Mechanik, die dezidiert eine Zeitenwende betreibt. Was zu tun ist, kommt bei dieser Theoretisierung noch nicht heraus.

Versichern wir uns daher kurz unserer Progression, um eventuell Progressivität zu finden: die invektivengetriebene Tour-de-force hat uns von einer Philosophie der Dummheit, zur Kulturanthropologie, Vulgärpsychologie, Geschlechtertheorie des Faschismus, zu den Niederungen des Horrors, Catchens, der Haaranalyse transportiert. Trump ist überall anschließbar; zugleich fällt auf, dass jede Hermeneutik vor allem die strukturelle Offensichtlichkeit und Anti-Komplexität des Objekts erschöpfend verdeutlicht; ,Interpretation“ ist wie befunden Mangelware. Treffen einzelne Beleidigungen und Theoreme, behauptet das Phänomen dennoch mit furios-simpler Resilienz seinen Stammplatz in diesem wasteland der Theoretisierbarkeit. Kritik säuft ab und verdorrt, auch weil aus Sicht des ,Systems Trump “ unsere Ansätze in den Worten des Kognitionswissenschaftlers Steven Pinker ja nur „outlandish“ sind. ${ }^{11}$ The barron land ist eine wüste Landschaft, in der es für outlander schwer möglich ist, Sinnstiftendes hinüber zu bringen, ohne totaler Erschöpfung anheim zu fallen. ${ }^{12}$ Abermals kommt der Befund unüberraschend: „Essentially linked to the inexhaustible, stupidity is also that which fatigues knowledge and wears down history“, lesen wir auf der ersten Seite von Stupidity (Ronell 2002: 1). Was Ronell 2002 wusste, erklärt Trump 2016 im prophetischen Futur einer kommenden Zeitenwende: „We gonna win so much you may even get tired of winning and you'll say please, please Mr. President, it's too much winning! We can't take it anymore!“ Chronik einer angekündigten Überhitzung und Erschöpfung des Erkenntnisgewinnens, an exhaustion of theory.

Es gibt keine Person/Persona Trump, die mit einer ,Hermeneutik des Verdachts“ (Ricœur) ideologiekritisch aufzuschlüsseln wäre; Ideologiekritik und die Frage nach dem „,cui bono“ erledigt The Don bereits selbst. „You'd have to show me the scientists [arguing climate change; S.S.] because they have a very big political agenda." Es ist ein diskursiv-symbolischer Klimawandel, der unter dem Stichwort ,Trump“ (oder ,Rechtsruck‘, oder ,Krise der Demokratie‘) primär zu erfühlen ist.

\footnotetext{
${ }^{11}$ Zitiert aus einem Tweet von Pinker zu der sog. ,Sokal Squared'-Affäre (vgl. Kafka 2018), die bestimmte Forschungsrichtungen durch gefälschte Forschungsartikel diskreditieren sollte: „Is there any idea so outlandish that it won't be published in a Critical/PoMo/Identity/,Theory “ journal? Helen Plucrose et al. submitted a dozen hoax papers to find out" (Tweet vom 03.10.2018, meine Hervorhebung). Ist die Kulturwissenschaft, der ich mich verbunden fühle, in diesem Sinn als generell ,outlandish/ausländisch ' bestimmt, kann ich das nur als Aufforderung zur Steigerung dieser Eigenschaft verstehen. So fuck it.

${ }^{12}$ Immerhin die Populärkultur scheint ihr Vergnügen am Zustand zu behalten: Das barren land USA ist primärer Topos in den Erfolgsfilmen Mad Max: Fury Road (2016) und The Bad Batch (2018). Ich führe sie auch deswegen an, weil beide Filme die Zukunft als dezidiert nicht-diskursiv imaginieren, in beiden wird kaum ein sinnvolles Wort gesprochen. Der wüsten Bildlichkeit entspricht jene ausgedörrte Sprache, die auch uns blüht.
} 
Über den notwendigen und bereits erfolgreichen Aktivismus gegen diesen Wandel hinaus sollten wir auch auf dem Papier anders agieren: die Arbeiter*innen der Kulturwissenschaften sind mithin aufgefordert, auch Meteorolog*innen zu werden. Wie bei diesen ändern weder Empörung noch Exegese etwas am Phänomen; die Vorhersage hilft aber dem Publikum, sich witterungsentsprechend anzuziehen, ggf. das Haus zu vernageln oder sich durch Evakuierung aus der Gefahrenzone zu bringen. Wir können also Ratschläge geben, wie man jenes barren land bewohnen kann (vgl. Strick 2015), das sich stetig vergrößert und ausweitet.

Als Modellfall sei abschließend Barron William Trump genannt, seit 2006 Einwohner der systemischen und symbolischen Wüstenei. Bei ihm finden wir ein Programm, das bei völliger Erschöpfung, Hilflosigkeit und dem Zwang, bleiben zu müssen, hilft. Die Theaterregisseurin Susann Neuenfeldt hat Barrons Figur zu einem Fixpunkt in ihrer Berliner Inszenierung [Donald Trump ist] Die Schneekönigin (2016) gemacht: eine Videoprojektion von Barrons Erschöpfung rahmte die hellsichtige Performance zur politischen Eiszeit auf Basis des Andersen-Märchens, herausgeschnitten aus der Fernsehübertragung von Trumps Wahlsieg am 9. November 2016, im Hilton Hotel von New York City, 22 Uhr Ortszeit. Fühlen wir mit diesem zehn Jahre alten Tired Trump, wie man im barren land wach(sam) bleibt:

$\mathrm{Zu}$ Vaters Siegesrede schwankt Barron hin und her, seine Augen suchen nach Fixpunkten; Scheinwerfer, Kameras, Gesichter, Punkte in der Menge, auf dem Boden. Er braucht Halt. Gegen die Erschöpfung führt Barron Stellvertreterkriege mit seinem Körper, ein Räuspern, Schniefen, er pustet sich die Haare aus dem Gesicht. Er kratzt sich am Ohr. Die Angriffe der Müdigkeit und Erschöpfung sind stärker als der siegreich lärmende Donald neben ihm: Barron hört nicht das „tremendous potential of this country“, von dem Vater spricht, denn das Gähnen durchzuckt seinen Körper. Er presst die Augen zu, die Lippen aufeinander und behält seine Not nach Sauerstoff oder den Schrei in der geschlossenen Mundhöhle. Er werde die Infrastruktur dieses Landes wieder aufbauen, verspricht Vater, und Barron fällt beinahe um; nur die im Reflex aufgerissenen Augen holen ihn zurück in diese seltsame Realität. Er steht auf der Bühne, ungleicher Gegenpart zum anderen, Vize“ Mike Pence (rechterhand). Die Gewerke der Polit-Show sieht er deutlich: Markierungen auf dem Boden, Kabel, Rückseite der Kulisse, Teleprompter - Halterungen für sein schwindendes Bewusstsein. „We will finally take care of our veterans", tönt Vater, und Veteran Barron zeigt offen sein Gähnen. Der sausende Zeigefinger punktiert die Luft; Barron richtet sich kurz auf wie eine Marionette, und fällt zurück in jene ,languid posture“ (Nabokov 1970: 190) der Kindheit, die Nabokov in Lolita beschreibt. Vater reckt zum Abschluss die Faust des Revolutionärs; Barron rollt die Augen, zieht die Nase hoch und wedelt mit den Armen.

„Flailing“ (Berlant 2018) ist der englische Begriff für Barrons erkenntnistheoretische Übung der Erschöpfung. Sie instruiert, wie man der rechten Dauerbefeuerung durch Autokraten-Muzak die eigene Aufmerksamkeit entziehen kann. Dieser Aufsatz hat flailing in der Theoriebildung versucht, um den Zustand der 
exhaustion performativ zu vollziehen, denn er ist von Vorteil: Sind wir erschöpft, kommen uns die hilflosen Strategien der Verdächtigung, der Empörung, der Richtigstellung, des Gegenbeweises, des zynischen oder sentimentalen recht-haben-Wollens abhanden. Einfache, wenn auch ungelenke Handlungen treten in den Vordergrund, Nebensächliches wird sichtbar und zentral, zahllose Fixpunkte bieten Halt, um aus der rechten Überwältigungslogistik auszusteigen, ohne ihr den Rücken kehren zu müssen: ,[...] the affective and political pressure of crisis disrupts both listening and reading: it forces skimming, flailing, jumping to conclusions, and trailing off into ellipsis, along with the collapse of the difference between obsession and distraction“ (Berlant 2017a). Ich verstehe das als Gewinn, denn flailing, im Umgang mit der Neuen Rechten, behält die Gewerke der populistischen Blendung im Auge, ohne sich an der Bedeutungsebene der Show abarbeiten zu müssen: wir wissen, was Lüge, was Manipulation, was Rassismus, was Metapolitik ist, die Protagonist*innen der rechten Zeitenwende reden in aller Öffentlichkeit von nichts anderem. Flailing verhindert, dass diese Unordnungen zum konturierten Polit-Objekt, zur Geschichtsmacht, zur Bedeutung hin verdichtet werden, wo sie in erster Linie affektiver Lärm und white kitsch sind. Flailing bringt jene andere Aufmerksamkeit, die die erschöpfte Leser*in nicht auf dem epistemological high ground positioniert, sondern den Schlaf abwehrt und so Kritik durch und als Geste im radikalen Präsens ermöglicht. Wir brauchen eine andere Wissenschaftspraxis, unterhalb der entschleiernden Hermeneutik (die schon immer alles verstanden hat) und kleiner als die ideologiekritische Intervention (die schon immer alles besser weiß). Sich der Trump Time nicht auszuliefern, der großen Aufmerksamkeitssteuerung und dem World Building des Obszönen zu entgehen, ist beim gegenwärtigen Klima ein wichtiger Schritt. Wie Barron sind die Erschöpften in diesem Sinn vielleicht schon post-Trump.

\section{Literatur}

Anonym: I am part of the resistance inside the Trump administration. In: New York Times (05.09.2018), https://www.nytimes.com/2018/09/05/opinion/trump-white-house-anonymousresistance.html (28.11.2018).

Berlant, Lauren: The queen of America goes to Washington City: Essays on Sex and Citizenship. Duke 1997.

Berlant, Lauren: Trump, or political emotions. In: The New Inquiry 5 (05.08.2016), https://thenewinquiry.com/trump-or-political-emotions/ (09.01.2019).

Berlant, Lauren: Big man. In: Social Text 19 (2017a), https://socialtextjournal.org/big-man/ (09.01.2019).

Berlant, Lauren: Humorlessness (Three Monologues and a Hairpiece). In: Critical Inquiry 43/2 (2017b): 305-340.

Berlant, Lauren: Genre flailing. In: capaciousjournal.com 1/2 (2018): 156-164.

Connell, Robert W./Messerschmidt, James W.: Hegemonic masculinity: Rethinking the concept. In: Gender \& Society 19/6 (2005): 829-859.

CBSN: Full video: Sarah Palin endorses Donald Trump. Youtube.com, 19.01.2016. https://www. youtube.com/watch?v=Mvlm3LKSlpU (06.03.2019).

Davies, Jude: Stupid white men: Toward a trans-atlantic politics of stupidity. In: REAL - Yearbook of Research in English and American Literature 23 (2007): 189-210. 
Dietze, Gabriele: Bluten, Häuten, Fragmentieren. Der Splatterfilm als Schwellenraum am Beispiel von The Texas Chainsaw Massacre und The Texas Chainsaw Massacre 2. In: Julia Köhne/Ralph Kuschke/Arno Meteling (Hg.): Splatter Movies. Essays zum modernen Horrorfilm. Berlin 2006: 89-100.

Dietze, Gabriele: Weiße Frauen in Bewegung: Genealogien und Konkurrenzen von Race-und Genderpolitiken. Bielefeld 2014.

Dyer, Richard: White. London/New York 1997.

Dyer, Richard: The President's hair (19.03.2018), https://www.the-platform.org.uk/2018/03/19/ president-donald-trump-hair/ (28.11.2018).

Frankenberg, Ruth: The Social Construction of Whiteness: White Women, Race Matters. Minneapolis 1993.

Gilman, Sander L./Thomas, James M.: Are Racists Crazy?: How Prejudice, Racism, and Antisemitism Became Markers of Insanity. New York 2016.

Girard, René: Das Heilige und die Gewalt. Frankfurt a. M. 1999.

Giroux, Henry A.: Donald Trump, Brett Kavanaugh and the path to neoliberal fascism (10.10.2018), https://www.salon.com/2018/10/10/donald-trump-brett-kavanaugh-and-thepath-to-neoliberal-fascism/ (28.11.2018).

Hawley, George: Making Sense of the Alt-right. New York 2017.

Johnson, Eric: Full transcript: WNYC's Kurt Andersen on Recode Media: The Spy magazine co-founder talks about branding Trump a ,short-fingered vulgarian' (01.11.2016), https:// www.recode.net/2016/11/1/13480986/kurt-andersen-wnyc-spy-donald-trump-recode-mediapodcast-transcript (28.11.2018).

Kafka, Alexander: ,Sokal squared‘: Is huge publishing hoax ,hilarious and delightful ‘ or an ugly example of dishonesty and bad faith? In: The Chronicle of Higher Education (03.10.2018), https://www.chronicle.com/article/Sokal-Squared-Is-Huge/244714 (28.11.2018).

Kavka, Misha: Trump, Fernsehen und das neue Camp. In: Zeitschrift für Medienwissenschaft 18 (2018): 149-168.

Kimball, Linda: Cultural marxism. In: American Thinker (15.02.2007), https://www.americanthinker.com/articles/2007/02/cultural_marxism.htm (28.11.2018).

Kimmel, Michael: Angry White Men: American Masculinity at the End of an Era. New York 2017.

Lee, Bandy X. u. a.: The Dangerous Case of Donald Trump: 27 Psychiatrists and Mental Health Experts Assess a President. New York 2017.

Limon, John: Stand-Up Comedy in Theory, or, Abjection in America. Durham 2000.

Manchester, Julia: Trump rode golf cart while G7 leaders walked through Sicily. In: The Hill (05.27.2017), http://thehill.com/homenews/administration/335424-trump-rode-golf-cart-while-g7-leaders-walked-through-siciliy (27.11.2018).

Mazer, Sharon: Donald Trump shoots the match. In: TDR: The Drama Review 62/2 (2018): 175200.

McKew, Molly: Brett Kavanaugh and the information terrorists are trying to reshape America. In: wired.com (10.03.2018), https://www.wired.com/story/information-terrorists-trying-to-reshape-america/ (28.11.2018).

McNeill, John: How fascist is Donald Trump? There's actually a formula for that. In: Washington Post (21.10.2016), https://www.washingtonpost.com/posteverything/wp/2016/10/21/how-fascist-is-donald-trump-theres-actually-a-formula-for-that/ (28.11.2018).

Malinowski, Bronislaw: Geschlecht und Verdrängung in primitiven Gesellschaften. Reinbek bei Hamburg 1962.

Nabokov, Vladimir: The Annotated Lolita [1955]. New York 1970.

Nyong'o, Tavia: Racial kitsch and black performance. In: The Yale Journal of Criticism 15/2 (2002): 371-391.

O'Toole, Fintan: Heroic Failure: Brexit and the Politics of Pain. London 2018.

Paschal, Olivia: Here are the most extraordinary quotes from Bob Woodward's book. In: The Atlantic (04.09.2018), https://www.theatlantic.com/politics/archive/2018/09/bob-woodwardbook-donald-trump/569293/ (28.11.2018). 
Rapley, Mark: The Social Construction of Intellectual Disability. Cambridge, Mass. 2004.

Ronell, Avital: Stupidity. Urbana-Champaign, Illinois 2002.

Roth, Angelica R.: Sen. Graham defends Kavanaugh: ,This is the most unethical sham since I've been in politics'. Youtube.com $(28.09 .2018)$, https://www.youtube.com/watch?v $=8 \mathrm{C} 9 \mathrm{i} 8 \mathrm{wd}-$ S-k (06.03.2019).

Rothkopf, Johanna: In honor of Donald Trump's 70th birthday, here are 70 ways jezebel has described Donald Trump. In: Jezebel.com (14.06.2016), https://theslot.jezebel.com/in-honorof-donald-trumps-70th-birthday-here-are-70-wa-1781899319 (28.11.2018).

Rubin, Gayle S.: The Traffic in Women: Notes on the "Political Economy" of Sex. In: Deviations: A Gayle Rubin Reader, Durham 2011: 33-65.

Schilling, Dave: Apocalypse now: Sarah Palin's bizarre Trump endorsement analyzed. In: The Guardian (20.01.2016), https://www.theguardian.com/us-news/2016/jan/20/sarah-palin-donald-trump-endorsement-speech-quotes (28.11.2018).

Schumacher, Elliot/Eskenazi, Maxine: A readability analysis of campaign speeches from the 2016 US presidential campaign. arXiv preprint arXiv (18.03.2016):1603.05739.

Seeßlen, Georg/Metz, Markus: Der Rechtsruck: Skizzen zu einer Theorie des politischen Kulturwandels. Berlin 2018.

Seeßlen, Georg: Trump!: POPulismus als Politik. Berlin 2017.

Strick, Simon: Alt-Right-Affekte: Provokationen und Online-Taktiken. In: Zeitschrift für Medienwissenschaften 10/2 (2018): 113-125.

Strick, Simon: Backlash: Trump oder das Lachen der Angst. In: Polar: Politik Theorie Alltag 21 (2016): 111-116.

Strick, Simon: How do you live? From construction to habitation. In: Critical Habitations (17.03.2015), https://criticalhabitations.wordpress.com/debate/how-do-you-live-from-construction-to-habitation (28.11.2018).

Theweleit, Klaus: Männerphantasien. Bd. 2: Männerkörper: Zur Psychoanalyse des weißen Terrors. Frankfurt a. M./Basel 1978.

Walz, Udo: Überkämmen oder Glatze? In: Tag24.de (24.01.2018), https://www.tag24. de/nachrichten/berlin-star-friseur-udo-walz-haarausfall-maenner-ueberkaemmen-oder-glatze-prominente-beispiele-431199 (28.11.2018).

Williams, Linda: Film bodies: Gender, genre, and excess. In: Film Quarterly 44/4 (1991): 2-13.

Young, Damon: 40 ways white people say, white people "without actually saying, white people". In: TheRoot.com (25.06.2018), https://verysmartbrothas.theroot.com/40-ways-white-peoplesay-white-people-without-actually-1827101126 (28.11.2018).

\section{Abbildungen}

Screenshot, Donald Trump victory speech (09.11.2016), https://www.youtube.com/watch?v= Qsvy10D5rtc (24.04.2019). 
Open Access Dieses Kapitel wird unter der Creative Commons Namensnennung 4.0 International Lizenz (http://creativecommons.org/licenses/by/4.0/deed.de) veröffentlicht, welche die Nutzung, Vervielfältigung, Bearbeitung, Verbreitung und Wiedergabe in jeglichem Medium und Format erlaubt, sofern Sie den/die ursprünglichen Autor(en) und die Quelle ordnungsgemäß nennen, einen Link zur Creative Commons Lizenz beifügen und angeben, ob Änderungen vorgenommen wurden.

Die in diesem Kapitel enthaltenen Bilder und sonstiges Drittmaterial unterliegen ebenfalls der genannten Creative Commons Lizenz, sofern sich aus der Abbildungslegende nichts anderes ergibt. Sofern das betreffende Material nicht unter der genannten Creative Commons Lizenz steht und die betreffende Handlung nicht nach gesetzlichen Vorschriften erlaubt ist, ist für die oben aufgeführten Weiterverwendungen des Materials die Einwilligung des jeweiligen Rechteinhabers einzuholen. 